Goldbach, Anne; Leonhardt, Nico

\title{
Elemente von Macht im Kontext einer inklusionssensiblen
}

\section{Hochschulentwicklung. Erfahrungen aus dem Hochschulprojekt der}

\section{Universität Leipzig 'Qualifizierung von Bildungs- und}

\section{Inklusionsreferent*innen in Sachsen' (QuaBIS)}

\author{
Grenzen.Gänge.Zwischen.Welten. Kontroversen - Entwicklungen - Perspektiven der
}

Inklusionsforschung. Bad Heilbrunn : Verlag Julius Klinkhardt 2022, S. 173-180

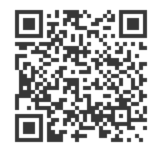

Quellenangabe/ Reference:

Goldbach, Anne; Leonhardt, Nico: Elemente von Macht im Kontext einer inklusionssensiblen

Hochschulentwicklung. Erfahrungen aus dem Hochschulprojekt der Universität Leipzig 'Qualifizierung von Bildungs- und Inklusionsreferent*innen in Sachsen' (QuaBIS) - In:

Grenzen.Gänge.Zwischen.Welten. Kontroversen - Entwicklungen - Perspektiven der

Inklusionsforschung. Bad Heilbrunn : Verlag Julius Klinkhardt 2022, S. 173-180 - URN:

urn:nbn:de:0111-pedocs-238290 - DOI: 10.25656/01:23829

https://nbn-resolving.org/urn:nbn:de:0111-pedocs-238290

https://doi.org/10.25656/01:23829

in Kooperation mit / in cooperation with:

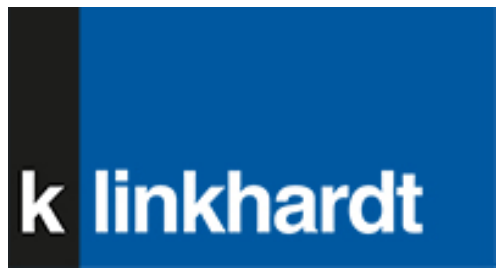

http://www.klinkhardt.de

\section{Nutzungsbedingungen}

Dieses Dokument steht unter folgender Creative Commons-Lizenz: http://creativecommons.org/licenses/by-nc-sa/4.0/deed.de - Sie dürfen das Werk bzw. den Inhalt unter folgenden Bedingungen vervielfältigen, verbreiten und öffentlich zugänglich machen sowie Abwandlungen und Bearbeitungen des Werkes bzw. Inhaltes anfertigen: Sie müssen den Namen des Autors/Rechteinhabers in der von ihm festgelegten Weise nennen. Dieses Werk bzw. der Inhalt darf nicht für kommerzielle Žwecke verwendet werden. Die neu entstandenen Werke bzw. Inhalte dürfen nur unter Verwendung von Lizenzbedingungen weitergegeben werden, die mit denen dieses Lizenzbedingungen weitergegeben werden,

Mit der Verwendung dieses Dokuments erkennen Sie die Nutzungsbedingungen an.

\section{Terms of use}

This document is published under following Creative Commons-License: http://creativecommons.org/licenses/by-nc-sa/4.0/deed.en - You may copy, distribute and transmit, adapt or exhibit the work in the public and alter, transform or change this work as long as you attribute the work in the manner specified by the author or licensor. You are not allowed to make commercial use of the work. If you alter, transform, or change this work in any way, you may distribute the resulting work only under this or a comparable license.

By using this particular document, you accept the above-stated conditions of

\section{Kontakt / Contact:}

peDOCS

DIPF | Leibniz-Institut für Bildungsforschung und Bildungsinformation Informationszentrum (IZ) Bildung

E-Mail: pedocs@dipf.de

Internet: www.pedocs.de

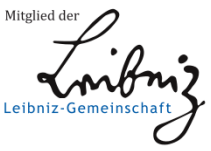




\section{Bernhard Schimek Gertraud Kremsner Michelle Proyer Rainer Grubich Florentine Paudel Regina Grubich-Müller (Hrsg.)}

\section{Grenzen.Gänge. Zwischen. Welten.}

\section{Kontroversen - Entwicklungen - Perspektiven der Inklusionsforschung}


Dieser Titel wurde in das Programm des Verlages mittels eines Peer-Review-Verfahrens aufgenommen. Für weitere Informationen siehe www.klinkhardt.de.

Bibliografische Information der Deutschen Nationalbibliothek Die Deutsche Nationalbibliothek verzeichnet diese Publikation in der Deutschen Nationalbibliografie; detaillierte bibliografische Daten sind im Internet abrufbar über http://dnb.d-nb.de.

2022.hg. () by Julius Klinkhardt.

Coverabbildung: Grafik: (C) Iris Kopera, Foto: kiyopayo/Adobe Stock.

Druck und Bindung: AZ Druck und Datentechnik, Kempten.

Printed in Germany 2022.

Gedruckt auf chlorfrei gebleichtem alterungsbeständigem Papier.

(c) (i)(3)(D) Die Publikation (mit Ausnahme aller Fotos, Grafiken und Abbildungen) ist veröffentlicht unter der Creative Commons-Lizenz: CC BY-NC-SA 4.0 International https://creativecommons.org/licenses/by-nc-sa/4.0/

ISBN 978-3-7815-5924-0 digital doi.org/10.35468/5924

ISBN 978-3-7815-2485-9 print 


\section{Inhalt}

Vorwort der Herausgeber*innen 9

Gertraud Kremsner, Bernhard Schimek und Michelle Proyer

Grenzen.Gänge.Zwischen.Welten.

Kontroversen - Entwicklungen - Perspektiven der Inklusionsforschung

\section{Kontroversen}

Georg Feuser

Die Bühne der Inklusion. Ein Prolog!

oder: Grenzgänge zwischen Welten

Mai-Anh Boger

Risse in der Landschaft der Inklusionsforschung -

Aktuelle Entwicklungen und offene Fragen

Sven Bärmig

Dialektik als Methode

Jan Jochmaring, Lena Bömelburg und Dirk Sponholz

Inklusive Berufsorientierung als Diskurs. Der ,scheinbare' Konsens:

gemeinsame Begriffe - unterschiedliche Ideen

Felix Kappeller

Prothetische Absenz, Articulating Gaps:

Repräsentationskritische Perspektiven auf visuelle Darstellungen

nicht an den Körper gefügter Prothesen

Jana York und Jan Jochmaring

Dilemmata einer inklusiven Arbeitswelt -

Menschen mit Behinderung zwischen Sondersystemen und

Gestaltungschancen einer Arbeitswelt 4.0

Juliane Gerland, Imke Niediek, Julia Hülsken und Marvin Sieger

Kontingenz von Differenzkonstruktionen in der inklusionsorientierten musikalischen Bildung am Beispiel des Umgangs

mit digitalen Musizier-Medien

Pierre-Carl Link

Zur Bedeutung einer befreiungspädagogischen Perspektive für die Inklusions- und Sonderpädagogik 
Katharina Hamisch und Robert Kruschel

Zwischen Individualisierungsversprechen und Vermessungsgefahr -

Die Rolle der Schlüsseltechnologie Künstliche Intelligenz in der inklusiven Schule

Felix Buchhaupt, Dieter Katzenbach, Deborah Lutz und Michael Urban

Zur Kontextualisierung der Inklusionsforschung

Lisa-Katharina Möhlen, Helena Deiß, Seyda Subasi Singh und Michelle Proyer

Lebenswelt(en der) Schule. Internationale Perspektiven zur Inklusion von

Schüler*innen mit Fluchterfahrungen. Eine Perspektive aus der Praxis

Thomas Hoffmann, Cathrin Reisenauer und Hendrik Richter

Helfen als individuelle Erfahrung und soziale Praxis zwischen

Deautonomisierung und Befähigung

\section{Entwicklungen}

Kathrin te Poel

Zur Bedeutsamkeit eigener schüler*innenbiografischer Erfahrungen von angehenden Lehrpersonen für den sich anbahnenden Lehrer*innenhabitus und seine Anerkennungsbezüge

Alina Quante und Oliver Danner

Grenzsetzungen bei Aufgabenbereichen von sonderpädagogischen und

allgemeinen Lehrkräften in inklusiven Settings

Edvina Bešić und Katharina Maitz

Das Boot: Eine Fluchtgeschichte -

Design-Based-Research in der Primarstufe

\section{Brigitte Kottmann}

Der Übergang von der Grundschule zur weiterführenden Schule als

(Soll-)Bruchstelle des Gemeinsamen Lernens

Anne Goldbach und Nico Leonhardt

Elemente von Macht im Kontext einer inklusionssensiblen

Hochschulentwicklung

Michaela Kaiser

Inklusionsbezogene Anforderungswahrnehmung -

Regulativ für (kunstpädagogische) Professionalisierung

Patrick Gollub, Silvia Greiten, Teresa Schkade und Marcel Veber

Schulpraktische Professionalisierung für den Umgang mit Heterogenität ein interdisziplinärer Blick aus hochschuldidaktischen Projekten 
Pierre-Carl Link, Cedric Steinert und Susanne Jurkowski

Implementierung von Inklusion als Querschnittsthema an der

Universität Erfurt durch das Kompetenz- und Entwicklungszentrum

für Inklusion. Inklusionsspezifische Professionalisierung der

Lehrer*innenbildung durch Team-Teaching, Fortbildung und

Online-Lernumgebung

\section{Perspektiven}

Timm Albers, Agnes Filipiak, Katja Franzen und Frank Hellmich

Kompetenzentwicklung im inklusiven Unterricht (KinU) -

eine internationale Perspektive

Katharina Maria Pongratz

Sehnsucht nach Bildung? Über den Einsatz systemischer Fragetechniken in der qualitativen Erwachsenenbildungs-/Weiterbildungsforschung bei Biografieträger*innen mit einer zugeschriebenen geistigen Behinderung

Laura Schwörer, Hannah van Ledden, Pia Algermissen und Mandy Hauser

Zusammenarbeit und Mediennutzung in einer

Partizipativen Forschungsgruppe

David Paulus, Patrick Gollub und Marcel Veber

Forschendes Lernen und Kasuistik. Grenzwelten und Zwischengänge

bezogen auf Reflexivität in der inklusionssensiblen Lehrer*innenbildung

Dietlind Gloystein und Ulrike Barth

Divers denken und handeln! - Theoretische Orientierungen und

Handlungsperspektiven für die Lehrkräftebildung

Ann-Christin Faix

Wie verändern sich die subjektiven Theorien von Lehramtsstudierenden über guten inklusiven Unterricht

Katja Baucke

Internationaler Vergleich als Reflexionsangebot. Eine explorative Studie zur Sicht von Hochschullehrenden auf schulische Inklusion in

Deutschland und Kanada

Bettina Amrhein, Benjamin Badstieber und René Schroeder

Zum Umgang mit als störend wahrgenommenen Handlungsweisen von

Schüler*innen in einem inklusionsorientierten Unterricht - Perspektiven

für die Lehrer*innenbildung (im Förderschwerpunkt emotionale und soziale Entwicklung) 
8 Inhaltsverzeichnis

Andrea Holzinger, Gerda Kernbichler, Silvia Kopp-Sixt, Mathias Krammer und Gonda Pickl

Profilierung für Inklusive Pädagogik (IP) im Lehramt der Primar- und

Sekundarstufe Allgemeinbildung

Timo Finkbeiner und Susanne Eibl

Kooperative Prozesse im technikbezogenen Unterricht 280

Autor*innenverzeichnis 287 
Anne Goldbach und Nico Leonhardt

\section{Elemente von Macht im Kontext einer inklusionssensiblen Hochschulentwicklung}

Erfahrungen aus dem Hochschulprojekt der Universität Leipzig ,Qualifizierung von Bildungs- und Inklusionsreferent*innen in Sachsen' (QuaBIS)

\section{Einleitung}

Hochschulen zeichnen sich durch viele Traditionen aus. Sie werden z.B. als Quelle von Wissen und als Innovationsmotor wahrgenommen. Zudem gilt die Hochschule als eine der beständigsten Institutionen überhaupt. Diese Beständigkeit zeigt sich jedoch auch in den unhinterfragten Traditionslinien standfester Barrieren, die durch selbstverständliche Leistungsmythen all denen einen Zugang verwehren, die nicht als ,fähig' kategorisiert werden (vgl. Powell 2016, 35ff.). Besonders deutsche Universitäten seien von einer „Aura der Exklusivität“ umgeben, so Alheit $(2014,196)$.

Ziel des QuaBIS-Projektes in Leipzig ist eine Veränderung dieser Machtstrukturen und damit eine veränderte Wissensproduktion, die zur Verankerung von inklusiver Bildung beiträgt.

Somit soll der Versuch gewagt werden, bisherige Grenzen zu überschreiten und Gänge aus einer abgeschotteten in eine bisher exklusive Welt zu ermöglichen. Im folgenden Beitrag sollen Aspekte eines alltäglichen Umgangs mit Machtfragen reflektiert und diskutiert werden, da diese unumgänglich zur gemeinsamen Arbeit gehören.

\section{Macht im Diskurs einer Pädagogik bei zugeschriebener Behinderung}

Macht ist „weder positiv noch negativ besetzt, sondern schlicht präsent. Eine Wertung kann erst im Einklang mit folgender Frage vorgenommen werden: Wie, durch wen und auf welche Weise kommt Macht zum Einsatz (und wirkt sich in weiterer Folge auf konkrete Personen aus) “ (Kremsner 2020, 10)? Dennoch wird schon mit Goffmans (1973) Beschreibungen zu ,Totalen Institutionen` für den 
Bereich der sogenannten Behindertenhilfe deutlich, dass Bewohner*innen solcher Institutionen über vergleichsweise sehr wenig Macht verfügen, gleichzeitig jedoch subtiler und direkter Machtausübung ausgesetzt sind (vgl. Kremsner 2020), worüber sich weder die Menschen mit zugeschriebener Behinderung noch die Professionellen bewusst sind (vgl. Kremsner 2017). Es gilt deshalb umso mehr, und insbesondere für inklusionssensible Entwicklungen auch innerhalb der exklusiven Institution Hochschule, eben diese Prozesse aufzudecken, um damit Reflexionsund Veränderungsmöglichkeiten zu schaffen.

Aufgrund der großen Vielfalt an theoretischen Zugängen zu Macht im Kontext einer Pädagogik bei zugeschriebener (geistiger) Behinderung ist es für die hier vorgenommene Auseinandersetzung zielführend, sich auf zwei machtspezifische Phänomene zu konzentrieren.

In einem ersten Fokus stehen demnach Othering-Prozesse, die zu einer kategorialen Zuschreibung und Konstruktion fundamentaler Andersheit (vgl. Boger \& Textor 2016) führen, wodurch ableistische Diskriminierung hervorgerufen wird. Solche Prozesse der Veranderung ${ }^{1}$ gründen in einer gesellschaftlich fest verwurzelten, hierarchischen und asymmetrischen Differenzordnung sowie damit verbundenen Herrschaftsverhältnissen (vgl. Riegel 2016). So kann bspw. mit Foucault davon ausgegangen werden, dass die Markierung einer Behinderung als Abweichung von der Norm erst durch den (wissenschaftlichen) Diskurs hervorgebracht wird und nicht per se besteht (vgl. Foucault, Seitter \& Konersmann 1996).

Der Begriff Othering beschreibt diesen Prozess des ,Different-Machens' (vgl. Castro Varela \& Dhawan 2005) und die damit verbundene Konstruktion der Anderen.

Für Menschen mit zugeschriebenen Behinderungen folgt aus dieser Zuschreibung häufig ein Ausschluss aus der allgemeinen normalisierten Gesellschaft und ein damit verbundener Einschluss in Hegemonialapparate der sogenannten Behindertenhilfe (vgl. Kremsner 2017), die, wie oben bereits beschrieben, selbst wiederum von asymmetrischen Beziehungen, Abhängigkeitsverhältnissen und Unterdrückung geprägt sind (vgl. u.a. Flieger 2015; Kremsner 2017; Kremsner 2020). Butler (2006) weist darauf hin, dass die Aufrechterhaltung und Reproduktion dieser Machtverhältnisse gesichert sind, weil Menschen, die immer wieder als ,Andere' markiert werden, sich selbst folglich auch als anders wahrnehmen. Im Sinne Spivaks kann dem hinzugefügt werden, dass ,im imperialen Macht- und Wissensdiskurs die Perspektiven und Stimmen von marginalisierten Anderen nicht gehört werden bzw. diese sprachlos gemacht werden" (Riegel 2016, 53) und sie dementsprechend auch wenig Möglichkeiten haben, auf ihre Situation aufmerksam zu machen, geschweige denn diese zu verändern.

1 Veranderung ist als deutscher Begriff zu Othering zu verstehen. 
Damit soll auf Subalternität als ein zweites machtspezifisches Phänomen (vgl. Spivak 2008) eingegangen werden. Subalterne (unterdrückte Gruppen) sind Menschen, die sich aufgrund ihrer Vorerfahrungen nicht trauen, zu sprechen, die nicht sprechen dürfen oder deren Stimme(n) einfach nicht gehört oder ernstgenommen werden (vgl. Kremsner 2017). Sprache wird dabei als ein Machtinstrument im Hinblick auf verschiedene Facetten theoretisch beleuchtet. Poststrukturalistisch lässt sich argumentieren, dass durch Sprache Realitäten erst hergestellt werden und Sprache somit ein großes Machtpotenzial innehat. Auch aus diskurstheoretischer Perspektive wird argumentiert, dass erst in (sprachlichen) Diskursen „implizit Dominanzordnungen, Normen und Ideologien zum Ausdruck gebracht [werden], die auf den hegemonialen Kontext und Herrschaftsverhältnisse verweisen“" (Riegel 2016, 28). Auch Butler (2006) beschreibt Sprache als ein Mittel der Macht zur Ausgrenzung und Herabsetzung von Menschen, gleichzeitig verweist sie jedoch auch darauf, dass eben jene performative Reproduktion von Differenzordnungen durch Sprache auch Potenzial hat, Widerstand und Veränderung hervorzurufen. Riegel (2016) verweist auf dieses Widerstandspotenzial beispielweise insofern, als dass „absichtliche Irritationen der Norm“ (ebd., 31) dazu beitragen können, eben solche Normalitätsgrenzen zu verschieben und zu verändern.

\section{Inklusionssensible Hochschulentwicklung}

So könnte beispielsweise inklusionssensible Hochschulentwicklung eine Möglichkeit darstellen, diese Performanz zu leisten, damit die Subalternität einzelner Menschen mit Behinderungserfahrung aufzuheben und ihre Stimme bedeutsamer werden zu lassen. Subjektperspektiven von Menschen mit Behinderungserfahrungen sollten ein ,Mehr' an gesellschaftlicher Sichtbarkeit und politischer Wirkmacht entfalten. Erst dann können gesellschaftlich gesetzte Standards, die Exklusion an sich erst reproduzieren (vgl. Kronauer 2002), stärker infrage gestellt und verändert werden.

Der Aufforderung zur Umsetzung inklusiver Hochschulentwicklung stellen sich die Hochschulen als Bildungs-, Ausbildungs- und Forschungseinrichtungen mit Bezug zur UN-Behindertenrechtskonvention zunehmend und bringen sich in diesen Diskurs allmählich ein: durch veränderte barrierefreie Studienbedingungen, inklusionsbezogene Hochschulforschungen, die Entwicklung verschiedener Hochschulstrategien/-aktionspläne und verschiedene andere Öffnungsprozesse (vgl. Dannenbeck, Dorrance, Moldenhauer, Oehme \& Platte 2016). Der Fokus der Maßnahmen in Deutschland beschränkt sich derzeit jedoch noch weitgehend auf das Feld Studium und Behinderung (vgl. Schuppener, Goldbach, Leonhardt, Langner \& Mannewitz 2020). Auch Hochschulen sind Räume pädagogischer Pra- 
xen. Diese „repräsentieren und konstituieren Machtverhältnisse, und sie werden von ihnen bestimmt. Darüber hinaus sind diese Praktiken in sozialen Räumen organisiert und gestalten soziale Räume“ (Bramberger \& Plaute 2018, 18).

Um der Hochschule inhärente Machtstrukturen und -verhältnisse offenzulegen, braucht es einen reflexiven Blick auf eben diese Differenzverhältnisse (vgl. ebd., 23).

Diversität und Partizipation werden im Kontext inklusiver (Hochschul)Bildung bislang primär auf der Ebene von Lernenden betrachtet (Heterogenität der Schüler*innenschaft, der Studierenden), weniger auf der Ebene der Lehrenden. Mit dem Anspruch einer Weiterentwicklung und diversitätsbewussten Öffnung von Hochschule sollte der Blick jedoch auch darauf gelenkt werden, welche Rolle beispielsweise Menschen mit Behinderungserfahrungen als Wissensvermittler*innen und damit Produzent*innen im akademischen Raum im Rahmen von Lehr- und Forschungstätigkeiten leisten können. Dieser Perspektiverweiterung widmet sich das Projekt QuaBIS, in welchem seit Mai 2019 u.a. an der Universität Leipzig sechs Menschen, die überwiegend vorher in einer Werkstatt für behinderte Menschen (WfbM) gearbeitet haben, zu sogenannten Bildungs- und Inklusionsreferent*innen in einer dreijährigen Qualifizierung ausgebildet werden. Sie sollen zukünftig Studierenden sowie Lehr-, Fach- und Führungskräften beispielhaft vermitteln, wie Inklusion und Diversität theoretisch zu verstehen sind, reflektiert werden können und praktisch funktionieren (vgl. Schuppener et al. 2020). Indem im Projekt QuaBIS sechs Menschen mit Behinderungserfahrungen eine Stimme im Kontext der Hochschullehre erhalten, tragen sie dazu bei, hochschultypische Machtstrukturen aufzubrechen und universitäre Machtansprüche durch in Gang gesetzte Empowermentprozesse besser zu verteilen (vgl. Noack 2003, 4f.). Ziel des Projektes ist es daher, eine Veränderung von Machtstrukturen und damit eine veränderte Wissensproduktion, die zur Verankerung inklusiver Bildung beiträgt und die von Butler (2006) beschriebenen ,Irritationen der Norm` befördern.

\section{Projekterfahrungen im Kontext machtvoller Strukturen}

Im Folgenden soll anhand von Aussagen der Bildungs- und Inklusionsreferent ${ }^{*}$ innen über eigene Machterfahrungen im Rahmen der Qualifizierung an der Hochschule reflektiert werden. Insbesondere auch darüber, inwiefern die derzeitigen Strukturen einer inklusionsorientierten Hochschulentwicklung eine Möglichkeit bieten, Machtverhältnisse zu hinterfragen und zu verändern. 


\subsection{Gewollt oder ungewollt stattfindende Othering-Prozesse}

Ein Teilnehmer der Qualifizierung schildert wahrgenommene Othering-Prozesse im Kontext der Hochschule wie folgt:

„Mein Empfinden ist, gerade in den Seminaren war es nur in einem Seminar, wo das Gefühl aufkam, dass man so klein ist, weil doch die meisten Studenten sowas Überlegenes hatten. Da hat man gemerkt, dass die einen Gymnasiumabschluss hatten und das haben die auch gezeigt, dass die so den Blick von oben herab haben. Es gibt aber auch andere Seminare, wo man das Gefühl hatte, dass man den gleichen Stand hatte."

Sehr deutlich kommt in diesem, aber auch in allen anderen noch folgenden Zitaten ein verinnerlichtes ,Anderssein“ zum Ausdruck (vgl. Kremsner 2020). Das Gefühl zu haben, „dass man so klein ist“, deutet auf vielfältige Erfahrungen von Unterdrückung hin. Die eigene Zuschreibung zur Gruppe der Anderen kann dabei als eine Folge der sozialen Wirkkraft hegemonialer Diskurse gedeutet werden (vgl. Butler 2006). Die als ,anders' Markierten bedienen sich dabei in gleicher Weise der Argumentationen derer, die sie als anders konstruieren (vgl. Riegel 2016). Ebenso wird ersichtlich, dass das eigene Anderssein erst in Bezug auf das Normalitäts-Dispositiv des Gymnasialabschlusses als relevant beschrieben und davon abgeleitet wird (vgl. ebd.). Trotz aller Kritik an der gesellschaftlich etablierten Praxis einer Unterscheidung in ,die Normalen' und ,die Anderen' ist zu hinterfragen, wie realistisch eine Auflösung von Normalitätsgrenzen im Sinne eines Transnormalismus tatsächlich ist (vgl. Boger 2019), oder ob es nicht vielmehr eine fortwährende Aufgabe ist, flexibelnormalistische Grenzen kritisch zu hinterfragen und anzupassen. So wird am oben genannten Zitat genauso deutlich, dass bestehende Differenzen nicht zwangsläufig zu einer wahrgenommenen Veranderung führen, sondern ein gemeinsames Studieren auf Augenhöhe ebenso möglich ist.

Eine Teilnehmerin beschreibt in ihren Gedanken über wahrgenommene Machterfahrungen im Bereich der Hochschule:

„Bei den jüngeren Studenten merkt man auch, dass die versuchen, ihre Macht auszuloten, unbewusst. Wenn die mich mit Dingen überbieten können, wenn die was sagen, wo ich dann nicht mitreden kann, weil ich kein Verständnis dafür hab.“

Neben dem auch hier deutlich werdenden verinnerlichten eigenen Anderssein verweist sie auf die von ihr wahrgenommene asymmetrische Beziehung und damit verbundene Machtverhältnisse zwischen „den jüngeren Studenten“ und ihr selbst als Bildungs- und Inklusionsreferentin. Sie erlebt, dass dieses Machtungleichgewicht zur Abgrenzung von ihr als ,Andere“, die scheinbar „kein Verständnis dafür“ hat, genutzt wird. Hierin verweist sie auch auf das Machtpotenzial von nicht angemessener Sprache, durch welche Ausgrenzung und damit Othering erfolgt. 


\section{Anne Goldbach und Nico Leonhardt}

\subsection{Erfahrungen mit Subalternität oder deren Auflösung}

In einigen Aussagen der Bildungs- und Inklusionsreferent*innen werden Erfahrungen mit Subalternität deutlich, die sowohl im derzeitigen Kontext der Qualifizierung an der Hochschule sichtbar werden als auch ihren Ursprung in früheren biografischen Erfahrungen haben. In Bezug auf Subalternität im Hochschulkontext sagt eine Teilnehmerin:

„Ich hab's halt schon gemerkt, dass einige Dozenten schon zeigen, dass sie hier Macht haben und mir zeigen: Da ist dein Platz, du hast in dem Sinne gar nichts zu sagen."

Auf der anderen Seite weist sie jedoch auch darauf hin, dass es ungewohnt ist und zum Teil eine Überforderung darstellt, plötzlich gehört und geachtet zu werden:

„Das Krasse war für mich, wo Saskia [Professorin im Fachbereich] mich als Kollegin hingestellt hat. Das hat noch nie jemand zu mir gesagt. Ich kannte das nicht, sonst hatte ich immer jemanden, der höher über mir stand und mich das hat auch spüren lassen und hier wird man so gleich behandelt. Ich hab auch Angst, den Anforderungen hier nicht gerecht zu werden, weil wenn mich Studentinnen fragen, wo ich mich machtlos fühle, wo ich nicht weiß, wie ich reagieren soll.“

In diesen Aussagen wird deutlich, dass sich durch die Erfahrungen von performativer Unterdrückung ein subalternes Selbstbild entwickeln kann (vgl. Butler 2006; Kremsner 2017; Spivak 2008), welches dazu führt, dass die Verschiebung bisheriger Grenzen bzw. Ordnungen gleichzeitig mit einem Moment der Ohnmacht einhergehen kann und Zugehörigkeit möglicherweise als brüchig wahrgenommen wird.

\section{Fazit/Ausblick}

Die untersuchten Zitate haben gezeigt, dass Differenzwahrnehmungen und eine damit verbundene Zuschreibung als ,Andere* $r^{*}$ eine Bedeutung für die erlebte Praxis im inklusionsorientierten Hochschulprojekt QuaBIS haben. Vor dem Hintergrund dessen, dass ohnehin zu hinterfragen ist, ob die Auflösung von Normalitätsgrenzen im Sinne des Transnormalismus möglich ist, sollte viel eher versucht werden, die Bedingungen herauszuarbeiten, die dazu führen können, dass Differenzen keine Otheringprozesse auslösen, sondern anerkennendes Miteinander ermöglichen. Die Zugehörigkeit zu einem bisher exklusiven System wie der Hochschule allein garantiert noch keine reale Verschiebung von Grenzen bzw. Ordnungen. Um diese Gänge über Grenzen hinweg zu durchschreiten, bedarf es viel mehr einer Reflexion der genannten Machtmechanismen. 
Es wurde deutlich, dass Sprache dabei als Mittel der Macht fungieren kann, um Ausgrenzung zu produzieren und Beteiligung zu verwehren. Butler sieht jedoch in der Performation auch eine Möglichkeit für Veränderung und Widerstand, durch performative „Irritation der Norm, der bewussten Verschiebung von Grenzen oder der Inszenierung von Zugehörigkeiten bzw. Nicht-Zugehörigkeiten bis hin zu Veränderungen vorherrschender Ordnungen“" (Riegel 2016, 31).

Durch die Beteiligung der Bildungs- und Inklusionsreferent*innen sollen eben solche Veränderungen angestoßen und reflektiert werden. Eine Aussage eines weiteren Teilnehmers zeigt, dass die Möglichkeit einer solchen (empowernden) Grenzverschiebung durchaus wahrgenommen wird:

„Das ist emotional die Uni. So wie ich das ganze [als] Komplex angucke, dann ist sie richtig machtvoll. Dann würd ich das so sehen, das ist ein Komplex. Eh ... die Fakultät ... und die Fakultät ... wo ich das Projekt, Gemeinsam Forschen ${ }^{2}$ gemacht hatte, ich habe dann irgendwie, irgendwann gemerkt, am ersten Tag: Uni: ,Wow, das ist ein riesen Teil!' Ich habe mich dann selbst - mich als Macht empfunden ... Wenn ich mir den ganzen Bereich der Universität angucke. Sie strahlt mir in dem Blickwinkel, strahlt genau dasselbe aus wie bei uns. Das wirkt bei uns mächtig und daraufhin strahlen wir diese selbe Macht aus, wie die Universität."

\section{Literatur}

Alheit, P. (2014): Die Exklusionsmacht des universitären Habitus. Exemplarische Studien zur „neuen deutschen Universität“. In: Ricken, N., Koller, H.-Ch. \& Keiner, E. (Hrsg.): Die Idee der Universität - revisited. Wiesbaden, 195-208.

Boger, M.-A. (2019): Theorien der Inklusion. Die Theorie der trilemmatischen Inklusion zum Mitdenken. Münster.

Boger, M.-A. \& Textor, A. (2016): Das Förderungs-Stigmatisierungs-Dilemma - Oder: Der Effekt diagnostischer Kategorien auf die Wahrnehmung durch Lehrkräfte. In: Amrhein, B. (Hrsg.): Diagnostik im Kontext inklusiver Bildung - Theorien, Ambivalenzen, Akteure, Konzepte. Bad Heilbrunn, 79-97.

Bramberger, A. \& Plaute, W. (2018): Bildungstheoretische Begründung einer inklusiven Hochschule. In: Harter-Reiter, S., Plaute, W. \& Schneider-Reisinger, R. (Hrsg.): Inklusive Hochschule. Diskursbausteine offener Hochschulbildung aus Theorie, Praxis und Forschung. Innsbruck, 17-24.

Butler, J. (2006): Hass spricht: Zur Politik des Performativen. Frankfurt am Main.

Castro Varela, M. d. M. \& Dhawan, N. (2005): Postkoloniale Theorie. Eine kritische Einführung. Bielefeld.

Dannenbeck, C., Dorrance, C., Moldenhauer, A., Oehme, A. \& Platte, A. (2016): Inklusionssensible Hochschule. Zur Einführung in diesen Band. In: Dannenbeck, C., Dorrance, C., Moldenhauer, A., Oehme, A. \& Platte, A. (Hrsg.): Inklusionssensible Hochschule. Grundlagen, Ansätze und Konzepte für Hochschuldidaktik und Organisationsentwicklung. Bad Heilbrunn, 9-21.

Flieger, P. (2015): Verteilt Leicht Lesen die Macht neu? In: Candussi, K. \& Fröhlich, W. (Hrsg.): Leicht Lesen. Der Schlüssel zur Welt. Wien/Köln \& Bonn, 143-154.

Foucault, M., Seitter, W. \& Konersmann, R. (1996): Die Ordnung des Diskurses. Frankfurt am Main.

2 Partizipatives Forschungsprojekt (2009-2011) an der Universität Leipzig (ESF) 


\section{Anne Goldbach und Nico Leonhardt}

Goffman, E. (1973): Asyle. Über die soziale Situation psychiatrischer Patienten und anderer Insassen. Frankfurt am Main.

Kremsner, G. (2017): Vom Einschluss der Ausgeschlossenen zum Ausschluss der Eingeschlossenen. Biographische Erfahrungen von so genannten Menschen mit Lernschwierigkeiten. Bad Heilbrunn.

Kremsner, G. (2020): Gewalt und Machtmissbrauch gegen Menschen mit Lernschwierigkeiten in Einrichtungen der Behindertenhilfe. In: Teilhabe, 59, 10-15.

Kronauer, M. (2002): Exklusion. Die Gefährdung des Sozialen im hoch entwickelten Kapitalismus. Frankfurt am Main/New York.

Noack, W. (2003): Wir müssen uns den Machtverhältnissen in der sozialen Arbeit stellen! In: Theorie und Praxis der Sozialen Arbeit, 54, H.6, 4-10.

Powell, J. J. W. (2016): Von Ableismus zur universal design Universität. In: Dannenbeck, C., Dorrance, C., Moldenhauer, A., Oehme, A. \& Platte, A. (Hrsg.): Inklusionssensible Hochschule. Grundlagen, Ansätze und Konzepte für Hochschuldidaktik und Organisationsentwicklung. Bad Heilbrunn, 34-51.

Riegel, Ch. (2016): Bildung - Intersektionalität - Othering. Pädagogisches Handeln in widersprüchlichen Verhältnissen. Bielefeld.

Schuppener, S., Goldbach, A., Leonhardt, N., Langner, A. \& Mannewitz, K. (2020): Inklusion inklusiv vermitteln: Menschen mit Behinderungserfahrungen als Lehrende an der Hochschule. In: Schneider-Reisinger, R. \& Oberlechner, M. (Hrsg.): Diversitätssensible PädagogInnenbildung in Forschung und Praxis: Utopien, Ansprüche und Herausforderungen. Leverkusen-Opladen, 108117.

Spivak, G. C. (2008): Can the Subaltern Speak? Postkolonialität und subalterne Artikulation. Wien. 